\title{
Hybrid damper with stroke amplification for damping of offshore wind turbines
}

\section{Brodersen, Mark L.; Høgsberg, Jan}

Published in:
Wind Energy

Link to article, DOI:

10.1002/we.1977

Publication date:

2016

Document Version

Peer reviewed version

Link back to DTU Orbit

Citation (APA):

Brodersen, M. L., \& Høgsberg, J. (2016). Hybrid damper with stroke amplification for damping of offshore wind turbines. Wind Energy, 19(12), 2223-2238. https://doi.org/10.1002/we.1977

\section{General rights}

Copyright and moral rights for the publications made accessible in the public portal are retained by the authors and/or other copyright owners and it is a condition of accessing publications that users recognise and abide by the legal requirements associated with these rights.

- Users may download and print one copy of any publication from the public portal for the purpose of private study or research.

- You may not further distribute the material or use it for any profit-making activity or commercial gain

- You may freely distribute the URL identifying the publication in the public portal

If you believe that this document breaches copyright please contact us providing details, and we will remove access to the work immediately and investigate your claim 


\title{
HYBRID DAMPER WITH STROKE AMPLIFICATION FOR DAMPING OF OFFSHORE WIND TURBINES
}

\author{
MARK L. BRODERSEN ${ }^{(1)}$ AND JAN HØGSBERG ${ }^{(1)}$ \\ (1) DEPARTMENT OF MECHANICAL ENGINEERING, TECHNICAL UNIVERSITY OF DENMARK, \\ DK-2800 KGS. LYNGBY, DENMARK
}

\begin{abstract}
The magnitude of tower vibrations of offshore wind turbines is a key design driver for the feasibility of the monopile support structure. A novel control concept for the damping of these tower vibrations is proposed, where viscous type hybrid dampers are installed at the bottom of the wind turbine tower. The proposed hybrid damper consists of a passive viscous dash-pot placed in series with a load cell and an active actuator. By integrated force feedback control of the actuator motion the associated displacement amplitude over the viscous damper can be increased compared to the passive viscous case, hereby significantly increasing the feasibility of viscous dampers acting at the bottom of the wind tower. To avoid drift in the actuator displacement a filtered time integration of the measured force signal is introduced. Numerical examples demonstrate that the filtered time integration control leads to performance similar to that of passive viscous damping and substantial amplification of the damper deformation without actuator drift.
\end{abstract}

\section{INTRODUCTION}

The monopile foundation is currently the most used support structure for shallow water offshore wind turbines. Compared to a jacket type foundation, the monopile has a simple and relatively cost-effective design, which on the other hand also makes it prone to wave loading. It is primarily a combination of the two lowest tower modes, the side-side mode and the fore-aft mode as seen in figure 1, which are excited by the waves. Especially waves misaligned with the wind direction can cause large fatigue damage due to the absence of aerodynamic damping in the direction lateral to the wind $[1,2]$. In the near future the majority of new offshore wind turbines will be taller and/or positioned at deeper waters, whereby the natural frequencies of the side-side mode and the fore-aft mode will be lowered. The natural frequencies will then move closer to the primary excitation frequency of the waves, causing resonant excitation to be more frequent. This may render the monopile support structure unfeasible. A means for increasing the feasibility of the monopile support structure, would be to add external damping to the critical modes of the wind turbine structure.

Several concepts have been proposed for structural control of tower vibrations of offshore wind turbines. Passive resonant damper concepts like e.g. a pendulum absorber or a tuned mass or liquid damper are well established concepts in structural control, and therefore widely used by the wind turbine manufactures for vibration control of offshore wind turbines [3, 4, 5, 6, 7]. A resonant damper like a pendulum absorber has the potential to achieve very high damping ratios, though in order for the absorber to be effective, large mass and space at the top of the wind turbine is required. As wind turbines become larger and located at larger water depths these mass and 


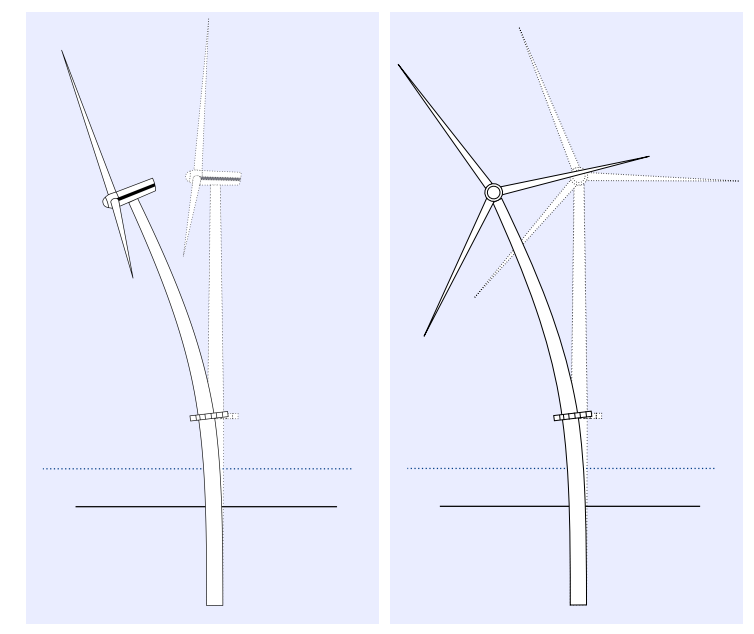

FiguRE 1. Fore-aft mode and side-side mode of a fixed offshore wind turbine

space requirements will increase and thereby limit the feasibility of pendulum type absorbers even further. Active concepts such as individual pitch control or active generator torque control has also been proposed for control of the side-side vibrations, see [8,9]. These concepts are relatively easy to integrate in a standard rotor-nacelle-assembly unit, though they may increase loading on other components such as the gear-box or the pitch actuators. More recently a passive concept has been proposed in [10], where dampers are installed inside the tower to act on the local curvature of the corresponding tower bending deformation. This concept utilizes stroke amplifying braces in order to amplify the relative motion of the tower, though the amplification is inherently limited for passive damping devices. The specific concept of using toggle-brace damping systems for wind turbines has recently been covered by the patent [11].

In the present paper it is proposed to install dampers inside the bottom of the tower by means of the viscous hybrid damper concept introduced in [12]. The hybrid damper concept includes a pure viscous dash-pot placed in series with both a force sensor and an active actuator. The actuator motion is controlled using a decentralized collocated control algorithm based on a filtered Integral Force Feedback (IFF) from the force sensor. In this paper a slightly modified controller scheme, without the filter time constant, is used. Eliminating the filter time scale from the feedback equation, the pure IFF scheme, introduced in $[13,14]$ for control of truss structures using piezoelectric transducers, is recovered. Among other applications the IFF format has also successfully been applied for control of cable stayed bridges $[15,16]$ and for control of large space structures [17]. In the present hybrid damper concept appropriate tuning of this IFF controller scheme introduces amplified motion of the structure locally at the damper position, leading to significantly larger stroke over the viscous dash-pot. Thus, the stroke amplification obtained by toggle-brace systems in $[10,11]$ is in the present case realized by active control. Since the generated hybrid damper force is in phase with the associated damper velocity the controller scheme is theoretically unconditionally stable in an idealized environment without time delays and actuator saturation. 
(a)

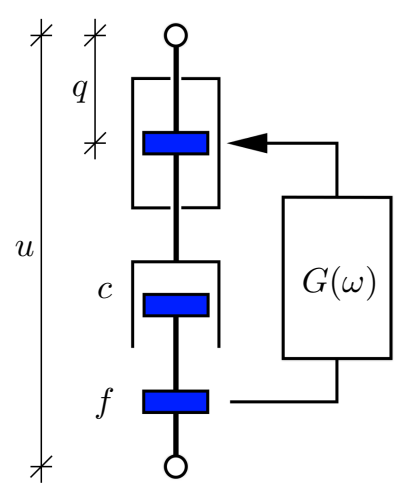

(b)

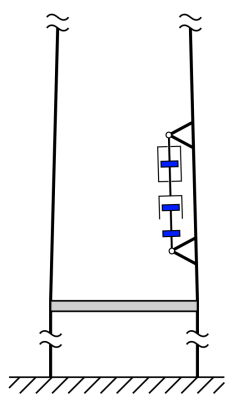

(c)

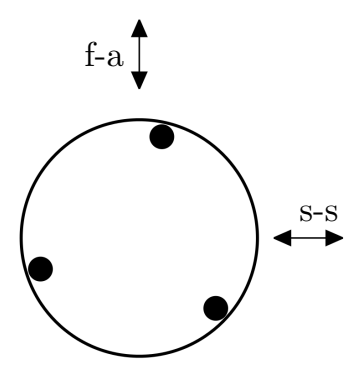

Figure 2. (a) Hybrid damper concept and its installation in the lower section of a wind turbine tower: (b) side view and (c) top view.

\section{The Hybrid Damper Concept}

The hybrid damper concept is illustrated in figure 2(a). It consists of a viscous dash-pot with viscous damping parameter $c$ in series with a load cell, measuring the damper force $f$, and an active actuator with piston motion $q$. The motion over the hybrid damper is given by $u$ whereby the measured force produced by the viscous dash-pot is given as

$$
f(t)=c(\dot{u}(t)-\dot{q}(t)) .
$$

The displacement of the actuator piston $q(t)$ is determined by an Integral Force Feedback (IFF) scheme [13], given as

$$
\dot{q}(t)=-g f(t),
$$

where $g$ is the control gain. For $g=0$ the passive limit without piston motion $q=0$ is recovered. In large scale structures, such as wind turbines, the desired damper reference signal $q$ may be realized by a hydraulic actuator with for example PID control of the piston motion, measured by a LVDT or an alternative displacement transducer. The implementation of active control strategies, and in particular IFF, is described in detail in [14]. The installation of the hybrid damper in a wind turbine tower is illustrated in figure $2(\mathrm{~b}, \mathrm{c})$. The direction of the fore-aft vibrations in the direction of the rotor is denoted by $\mathrm{f}$-a, while the direction of the side-side vibrations is denoted by s-s. In these, the two most critical tower modes with regards to wave excitation, the tower is primarily deformed in bending, and the damper should therefore be installed at the bottom of the tower in order to maximize modal interaction, as also illustrated in a side-view of the tower in figure 2(b). During operation the Rotor Nacelle Assembly (RNA) will change orientation following the direction of the wind. Thus, three or more dampers are needed in the circumferential direction to form a symmetric layout that leads to efficient damping of both the fore-aft mode and the side-side mode, independent of the specific orientation of the RNA. The three-damper layout, as illustrated by the top-view of the tower in figure 2(c), is also applied for the toggle-brace systems in [10, 11]. 
2.1. Frequency domain analysis. The hybrid damper characteristics are now investigated in the frequency domain. Harmonic solutions are assumed using the complex representations

$$
u(t)=\bar{u} \exp i \omega t \quad, \quad q(t)=\bar{q} \exp i \omega t \quad, \quad f(t)=\bar{f} \exp i \omega t
$$

with angular frequency $\omega$ and with amplitudes denoted by a bar. In the frequency domain the damper force in (1) is given by

$$
\bar{f}=i \omega c(\bar{u}-\bar{q})
$$

The corresponding feedback equation in (2) now appears as

$$
i \omega \bar{q}=-g \bar{f}
$$

which can be rewritten in terms of the transfer function

$$
G(\omega)=\frac{-g}{i \omega}
$$

to establish the feedback relation $\bar{q}=G(\omega) \bar{f}$ shown in figure $2($ a). The relation between damper force $\bar{f}$ and the displacement $\bar{u}$ of the hybrid damper is obtained by eliminating $\bar{q}$ between (4) and (5). This gives the force relation

$$
\bar{f}=i \omega c H \bar{u}
$$

where the transfer function $H$ in the present case is given as the constant

$$
H=\frac{1}{1-\nu} \text {. }
$$

with non-dimensional gain

$$
\nu=c g \text {. }
$$

In (8) the transfer function $H$ modifies the transfer relation of the hybrid damper relative to the pure viscous damper, which in the following will be used as benchmark for assessing the performance of the proposed hybrid damper concept. In order to have positive damping it is required that $H>0$, thereby invoking the limit $\nu<1$. The phase $\varphi$ of the damper force is defined by the relation $\tan \varphi=\operatorname{Im}[H] / \operatorname{Re}[H]$, which for $\nu<1$ gives $\varphi=0$. Thus, for the pure IFF controller the damper force is fully in phase with damper velocity, and the controller is therefore unconditionally stable for $\nu<1$. The amplification of the displacement over the viscous dash-pot relative to the passive case with $q=0$ is given by the magnitude $|H|$ of the transfer function

$$
|H|=\left|\frac{\bar{u}-\bar{q}}{\bar{u}}\right|=\left|\frac{1}{1-\nu}\right| .
$$

Thus, for $0<\nu<1$ the displacement of the viscous dash-pot is amplified relative to the passive case with $q=0$, whereas for $\nu=0$ the pure viscous case is recovered. In this way the hybrid damper can be used to increase the feasibility of installing a viscous damper in a flexible structure, such as a wind turbine, where the displacement over the damper is inherently small. 
2.2. Filtered integration. The hybrid damper implemented with the IFF controller, as given by the feedback relation in (6), has a pole at $s=i \omega=0$ in the Laplace domain. This makes it prone to drift in the actuator displacement, which again can lead to saturation of the actuator signal. In order to reduce this drift the actuator velocity is now integrated by a suitable filter. First, the actuator velocity is defined as an independent variable $v=\dot{q}$ and the feedback equation in (2) is therefore rewritten as

$$
v(t)=-g f(t)
$$

Subsequently, the actuator displacement $q$ is obtained by integration of the velocity $v$ through the second order filter

$$
\omega_{f} q(t)+\dot{q}(t)+\tau_{f} \ddot{q}(t)=v(t) .
$$

The first term with the corner frequency $\omega_{f}$ reduces drift and potential actuator saturation in the command displacement signal $q$. Note, that this is similar to the introduction of a forgetting factor in [13]. The last term in (12) with the filter time constant $\tau_{f}$ is added to compensate for the phase delay caused by the first term. The influence of this term will be demonstrated later in this section. Combining equation (11) and (12) gives

$$
\omega_{f} q(t)+\dot{q}(t)+\tau_{f} \ddot{q}(t)=-g f(t)
$$

In the frequency domain, obtained by assuming harmonic solutions as in (3), this equation can be written as

$$
\left(\omega_{f}-\omega^{2} \tau_{f}+i \omega\right) \bar{q}=-g \bar{f}
$$

whereby the modified transfer function $G(\omega)$ is given as

$$
G(\omega)=\frac{-g}{\omega_{f}-\omega^{2} \tau_{f}+i \omega}
$$

The previous pole at $i \omega=0$ for the pure IFF format is now replaced by the pair of complex conjugate poles

$$
s=i \omega=-1 /\left(2 \tau_{f}\right) \pm \sqrt{\omega_{f} / \tau_{f}} \sqrt{1 /\left(4 \omega_{f} \tau_{f}\right)-1}
$$

which reduces the actuator drift. Combining (14) with (4), the transfer function $H(\omega)$ defined in (7) is now given as

$$
H(\omega)=\frac{\omega_{f}-\tau_{f} \omega^{2}+i \omega}{\omega_{f}-\tau_{f} \omega^{2}+i \omega(1-\nu)}
$$

with real and imaginary parts

$$
\operatorname{Re}[H(\omega)]=\frac{\left(\omega_{f}-\tau_{f} \omega^{2}\right)^{2}+\omega^{2}(1-\nu)}{\left(\omega_{f}-\tau_{f} \omega^{2}\right)^{2}+\omega^{2}(1-\nu)^{2}} \quad, \quad \operatorname{Im}[H(\omega)]=\frac{\omega \nu\left(\omega_{f}-\tau_{f} \omega^{2}\right)}{\left(\omega_{f}-\tau_{f} \omega^{2}\right)^{2}+\omega^{2}(1-\nu)^{2}} .
$$

For $\omega_{f}, \tau_{f} \rightarrow 0$ the transfer function recovers the expression given in (8) for the pure IFF format. The frequency characteristics of $H(\omega)$ given in (17) are conveniently described in terms of the magnitude

$$
|H(\omega)|=\left|\frac{\bar{u}-\bar{q}}{\bar{u}}\right|=\sqrt{\frac{\left(\omega_{f}-\tau_{f} \omega^{2}\right)^{2}+\omega^{2}}{\left(\omega_{f}-\tau_{f} \omega^{2}\right)^{2}+\omega^{2}(1-\nu)^{2}}},
$$


which represents the amplification of the damper displacement, and by the phase angle $\varphi$ obtained from

$$
\tan \varphi=\frac{\operatorname{Im}[H(\omega)]}{\operatorname{Re}[H(\omega)]}=\frac{\omega \nu\left(\omega_{f}-\tau_{f} \omega^{2}\right)}{\omega^{2}(1-\nu)+\left(\omega_{f}-\tau_{f} \omega^{2}\right)^{2}} .
$$

It can be seen from (19) that the amplification of the displacement over the viscous dash-pot is in general reduced for non-negative filter parameters $\omega_{f}, \tau_{f}>0$, which unfortunately reduces the performance of the hybrid damper. However, by choosing $\omega_{f}$ and $\tau_{f}$ so that $\omega_{f}=\tau_{f} \omega_{0}^{2}$, where $\omega_{0}$ is the natural frequency of the structure, the magnitude and phase at this structural frequency $\omega_{0}$ becomes

$$
\left|H\left(\omega_{0}\right)\right|=\left|\frac{1}{1-\nu}\right| \quad, \quad \varphi\left(\omega_{0}\right)=0 .
$$

It is seen that the result for the pure IFF format in (8) is in fact recovered at structural resonance corresponding to $\omega=\omega_{0}$.

Figure 3 shows a plot of (a) the magnitude $|H(\omega)|$ as given in (19) and (b) the phase angle $\varphi$ obtained from (20). The non-dimensional gain value is $\nu=0.5$, while different values of $\omega_{f}$ and $\tau_{f}$ are chosen. The solid curves in the figure are for different non-negative values of $\omega_{f}=\tau_{f} \omega_{0}^{2}<\omega_{0}$, while the dashed curves represent the particular case with $\tau_{f}=0$ and different non-negative values of $\omega_{f}<\omega_{0}$. The values of $\omega_{f}$ are $\omega_{0} / 20$ (blue), $\omega_{0} / 8$ (red), $\omega_{0} / 5$ (green), and 0.00 (straight black). The latter case with $\omega_{f}=0$ corresponds to the case without filtered integration. For $\tau_{f}=0$ and $0<\omega_{f}<\omega_{0}$ (dashed curves) the magnitude is seen to be reduced to $|H(\omega)|<2$ compared to the case without filtered integration (straight black solid curve) with $|H|=2$. The reduction is largest for frequencies below the resonance frequency $\omega_{0}$. For the case with $\omega_{f}=\tau_{f} \omega_{0}^{2}$ (solid curves) the magnitude is reduced for the frequency intervals below and above the resonance frequency $\omega_{0}$, while around the resonance frequency $\omega=\omega_{0}$ the desired level of stroke amplification is retained. Thus, the amplification of the displacement of the damper is at its maximum at the structural resonance frequency where effective vibration damping is required.

The phase angle is plotted in figure 3(b). For $\tau_{f}=0$ (dashed curves) a phase lead $\varphi>0$ is observed compared to the pure viscous case (straight black solid curve). For the case with $\omega_{f}=\tau_{f} \omega_{0}^{2}$ (solid curves) a phase lead is observed below the resonance frequency $\omega_{0}$, while a phase $\operatorname{lag} \varphi<0$ is obtained for frequencies above $\omega_{0}$ with vanishing phase angle $\varphi=0$ at $\omega=\omega_{0}$. A phase lead $\varphi>0$ implies that the damper force acts ahead of the conjugated damper velocity, while a phase lag $\varphi<0$ corresponds to a damper force trailing velocity. The effect of a phase difference compared to pure viscous damping is discussed in [18], where it is demonstrated that a phase lead results in improved damper performance. Thus, by choosing the filter parameters from the equality $\omega_{f}=\tau_{f} \omega_{0}^{2}$ the drift of the actuator motion can be reduced, while retaining the desired displacement amplification at the natural frequency $\omega_{0}$ of the critical mode(s) of the wind turbine. In practice, the filter parameters should be chosen large enough to limit drift, but also small enough not to deteriorate the damping performance of the hybrid damper in the frequency range close to the structural frequency $\omega_{0}$. 
(a)

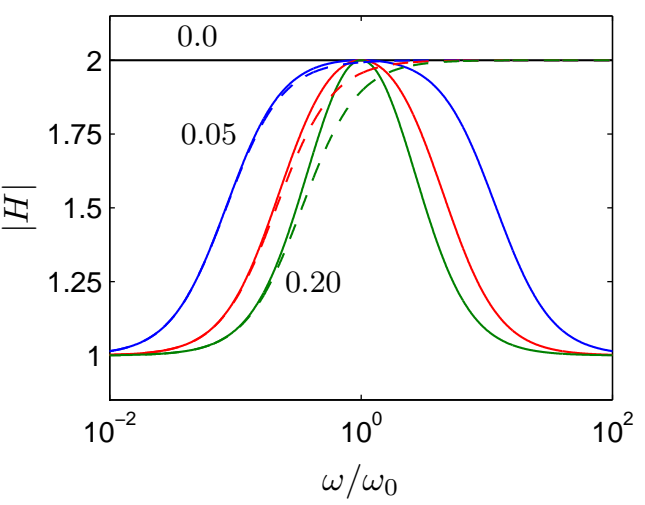

(b)

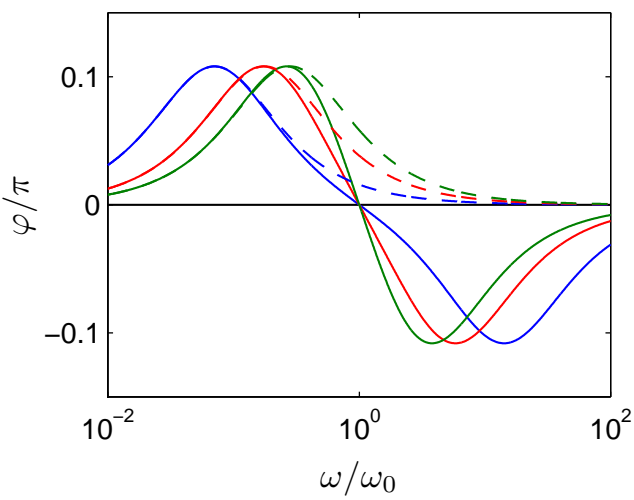

Figure 3. (a) Magnitude and (b) phase of frequency transfer function $H(\omega)$ for $\nu=0.5$ with $\tau_{f}=\omega_{f} / \omega_{0}^{2}$ (solid) and $\tau_{f}=0$ (dashed), and filter frequency $\omega_{f}=0.0$ (black), $\omega_{0} / 20$ (blue), $\omega_{0} / 8$ (red) and $\omega_{0} / 5$ (green).

\section{Properties OF Wind TURBINE AND DAMPERS}

The present section introduces a numerical wind turbine model for later use in the investigation of damping of tower vibrations, with particular emphasis on the installation of the hybrid dampers. The wind turbine model is the $5 \mathrm{MW}$ offshore wind turbine from the Offshore Code Comparison Collaboration study (OC3), see [19]. The wind turbine is supported by a monopile foundation, which is positioned at $20 \mathrm{~m}$ water depth with a penetration length of $36 \mathrm{~m}$ and connected to the wind turbine tower $10 \mathrm{~m}$ above Mean Sea Level (MSL). The tower is connected to the nacelle at $87.6 \mathrm{~m}$ above MSL, with the hub located at a height of $90 \mathrm{~m}$. Dampers are installed on the tower wall at the bottom of the tower with the upper point of attachment located $4 \mathrm{~m}$ above the bottom.

3.1. Linear beam model. Initially, the wind turbine is assumed to be at standstill, and the wind turbine and monopile are therefore represented by a linear discrete beam model, with the horizontal $y$-axis in the rotor direction, the horizontal $x$-axis lateral to the rotor direction and the vertical $z$-axis in the longitudinal tower direction. The beam model is three-dimensional with three translational displacements $\mathbf{u}_{i}^{T}=\left[u_{x i} u_{y i} u_{z i}\right]$ and three rotations $\varphi^{T}=\left[\varphi_{x i} \varphi_{y i} \varphi_{z i}\right]$ at element nodes $i=1$ and 2 . Thus, the displacements of beam element $j$ are described by the 12 degrees of freedom (dofs) contained in vector $\mathbf{u}_{j}^{T}=\left[\mathbf{u}_{1}^{T} \varphi_{1}^{T} \mathbf{u}_{2}^{T} \varphi_{2}^{T}\right]$, as shown in figure 4a. The equations of motion for the total number of structural dofs in $\mathbf{u}$ are given by

$$
\mathbf{M u ̈}+\mathbf{C} \dot{\mathbf{u}}+\mathbf{K u}=\mathbf{f}-\mathbf{f}_{d},
$$

where $\mathbf{M}, \mathbf{C}$ and $\mathbf{K}$ are the mass, damping and stiffness matrix, respectively, while $\mathbf{f}$ is the external load vector and $\mathbf{f}_{d}$ is the force vector representing the contributions from all hybrid dampers installed inside the tower. The stiffness matrix $\mathbf{K}$ consists of a contribution from the constitutive stiffness matrix of the tower and monopile, based on the complementary energy principle following [20], and a contribution from the soil stiffness, which is represented using linear springs. The mass matrix $\mathbf{M}$ represents the distributed mass and inertia of the tower and monopile, and the inertia and mass of the nacelle, rotor and blades which are lumped at the top of the tower. The 
damping matrix $\mathbf{C}$ is a proportional damping matrix that represents structural - and soil damping, and it is tuned to provide the two targeted tower modes (fore-aft and side-side) with a realistic damping ratio of $\zeta_{s t}=0.0115$ at standstill. The connection of the hybrid dampers to the tower are conveniently described by a connectivity vector $\mathbf{w}$ for each damper, whereby the damper force vector is given by the summation

$$
\mathbf{f}_{d}=\sum_{k=1}^{r} \mathbf{w}_{k} f_{k}
$$

where $r$ is the total number of dampers. Since the hybrid dampers are collocated the damper displacement $u_{k}$ of the $k^{\prime}$ th damper is determined as

$$
u_{k}=\mathbf{w}_{k}^{T} \mathbf{u}
$$

The connectivity vector $\mathbf{w}_{k}$ for the $k^{\prime}$ th damper has the same dimension as the number of dofs in the structural model, and it contains all zeros except at the dofs for the two adjacent nodes $n$ and $n+1$ where the dampers are attached in a circumferential configuration similar to that shown in figure 4c. The connectivity vector of the $k^{\prime}$ th damper can therefore be written as

$$
\mathbf{w}_{k}=\left[\begin{array}{llllllll}
0 & \ldots & 0 & \mathbf{w}_{k, n}^{T} & \mathbf{w}_{k, n+1}^{T} & 0 & \ldots & 0
\end{array}\right]^{T},
$$

where the two nodal connectivity vectors $\mathbf{w}_{k, n}$ and $\mathbf{w}_{k, n+1}$ associated with nodes $n$ and $n+1$ of the beam model represent the lower and upper points of the damper attachment inside the tower. The vectors are determined by computing the nodal load vector that imposes the damper forces at the two tower nodes $n$ and $n+1$. This gives

$$
\mathbf{w}_{k}^{n}=\left[\begin{array}{c}
\cos \left(\theta_{2}^{k}\right) \cos \left(\theta_{1}\right) \\
\sin \left(\theta_{2}^{k}\right) \cos \left(\theta_{1}\right) \\
-\sin \left(\theta_{1}\right) \\
-\sin \left(\theta_{2}^{k}\right) \sin \left(\theta_{1}\right) d_{1} / 2 \\
\cos \left(\theta_{2}^{k}\right) \sin \left(\theta_{1}\right) d_{1} / 2 \\
0
\end{array}\right] \quad, \quad \mathbf{w}_{k}^{n+1}=\left[\begin{array}{c}
-\cos \left(\theta_{2}^{k}\right) \cos \left(\theta_{1}\right) \\
-\sin \left(\theta_{2}^{k}\right) \cos \left(\theta_{1}\right) \\
\sin \left(\theta_{1}\right) \\
\sin \left(\theta_{2}^{k}\right) \sin \left(\theta_{1}\right) d_{2} / 2 \\
-\cos \left(\theta_{2}^{k}\right) \sin \left(\theta_{1}\right) d_{2} / 2 \\
0
\end{array}\right] .
$$

Here $\theta_{1}$ is the conic angle of the tower, while $d_{1}$ and $d_{2}$ are the diameters of the tower at the lower and upper points of damper attachment inside the tower, as shown in figure $4 \mathrm{~b}$. The angle $\theta_{2}^{k}$ represents the circumferential orientation of the $k^{\prime}$ th damper as seen in figure $4 \mathrm{c}$. The expressions for the two connectivity vectors in (26) only differ in terms of sign and magnitude of the lever arms $d_{1} / 2$ and $d_{2} / 2$.

3.2. Stability. Inserting the viscous relation for the damper force in (1) and the feedback relation as given by (13) into the equations of motion in (22) provides the governing closed-loop equations as

$$
\left[\begin{array}{cc}
\mathbf{M} & \mathbf{0} \\
\mathbf{0}^{T} & \tau_{f} \mathbf{I}
\end{array}\right]\left[\begin{array}{l}
\ddot{\ddot{u}} \\
\ddot{\mathbf{q}}
\end{array}\right]+\left[\begin{array}{cc}
\mathbf{C}+c \mathbf{W} \mathbf{W}^{T} & -c \mathbf{W} \\
\nu \mathbf{W}^{T} & (1-\nu) \mathbf{I}
\end{array}\right]\left[\begin{array}{c}
\dot{\mathbf{u}} \\
\dot{\mathbf{q}}
\end{array}\right]+\left[\begin{array}{cc}
\mathbf{K} & \mathbf{0} \\
\mathbf{0}^{T} & \omega_{f} \mathbf{I}
\end{array}\right]\left[\begin{array}{l}
\mathbf{u} \\
\mathbf{q}
\end{array}\right]=\left[\begin{array}{l}
\mathbf{f} \\
\mathbf{0}
\end{array}\right],
$$

where the dofs of the actuators are conveniently collected in the single column vector $\mathbf{q}^{T}=$ $\left[\begin{array}{lll}q_{1} & \ldots & q_{r}\end{array}\right]$, while the connectivity vectors are combined into a single matrix $\mathbf{W}=\left[\begin{array}{lll}\mathbf{w}_{1} & \ldots & \mathbf{w}_{r}\end{array}\right]$. Assuming that $\omega_{f}, \tau_{f}>0$ a non-negative energy functional for the system of equations of motion 
(a)

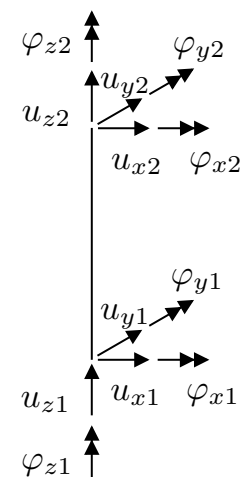

(b)

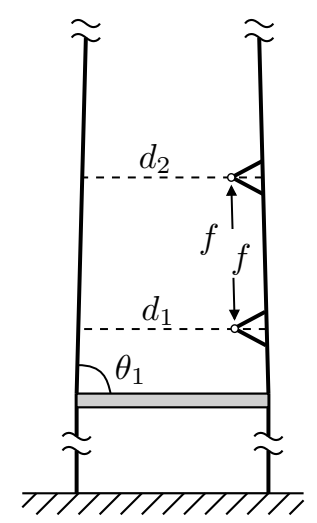

(c)

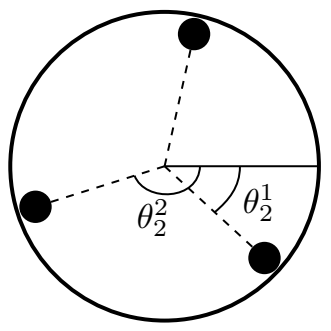

FIgURE 4. (a) 3D beam element and damper connectivity inside wind turbine tower: (b) side view and (c) top view.

in $(27)$ is formulated as

$$
V=\frac{1}{2} \dot{\mathbf{u}}^{T} \mathbf{M} \dot{\mathbf{u}}+\frac{1}{2} \frac{\tau_{f}}{g} \dot{\mathbf{q}}^{T} \dot{\mathbf{q}}+\frac{1}{2} \mathbf{u}^{T} \mathbf{K} \mathbf{u}+\frac{1}{2} \frac{\omega_{f}}{g} \mathbf{q}^{T} \mathbf{q} \geq 0 .
$$

The corresponding rate of this function is obtained from (27) and given by the expression

$$
\dot{V}=-\dot{\mathbf{u}}^{T} \mathbf{C} \dot{\mathbf{u}}-c\left(\dot{\mathbf{u}}^{T} \mathbf{W} \mathbf{W}^{T} \dot{\mathbf{u}}+\dot{\mathbf{q}}^{T} \dot{\mathbf{q}} \frac{1-\nu}{\nu}\right) .
$$

This rate of the functional is negative for $0 \leq \nu<1$. Thus, for $(\mathbf{u}, \dot{\mathbf{u}}, \mathbf{q}, \dot{\mathbf{q}}) \neq 0$ the energy $V$ is a Lyapunov function and the system in (27) is therefore asymptotically stable for $0 \leq \nu<1$ according to Lyapunov's second stability theorem [21].

3.3. Damper tuning. As demonstrated by Main and Krenk [22] the optimum tuning of supplemental viscous dampers can be estimated from a two-component representation, where the structural motion is expressed as a linear combination of the undamped mode shape $\mathbf{u}_{0}$ determined from the undamped eigenvalue problem

$$
\left(\mathbf{K}-\omega_{0}^{2} \mathbf{M}\right) \mathbf{u}_{0}=0,
$$

with natural frequency $\omega_{0}$, and the corresponding undamped mode shape vector $\mathbf{u}_{\infty}$ associated with fully rigid viscous dampers and governed by the modified eigenvalue problem

$$
\left(\mathbf{K}-\omega_{\infty}^{2} \mathbf{M}\right) \mathbf{u}_{\infty}=\mathbf{r}
$$

with frequency $\omega_{\infty}$. In (31) the force vector $\mathbf{r}$ contains the reaction forces from the rigid dampers on the structure. Following the procedure outlined in [22] the optimum viscous parameter for the hybrid damper is obtained by the relation

$$
c_{o p t} \omega|H(\omega)|=\frac{\omega_{\infty}^{2}-\omega_{0}^{2}}{\gamma^{2}}
$$

Here $|H(\omega)|$ is the amplitude of the transfer function given in (19), while

$$
\gamma^{2}=\mathbf{u}_{0}^{T}\left(\mathbf{W} \mathbf{W}^{T}\right) \mathbf{u}_{0}
$$


(a)

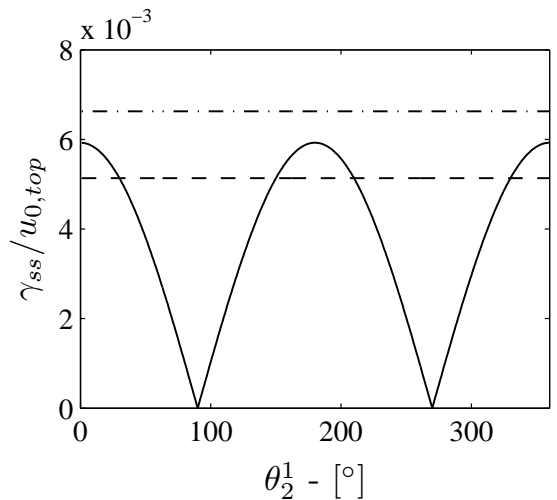

(b)

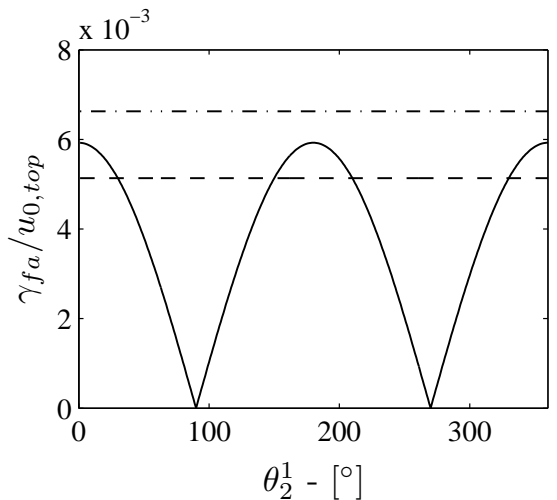

Figure 5. Modal damper displacement $\gamma$ scaled by the modal tower top displacement $u_{0, t o p}$ for (a) side-side and (b) fore-aft mode

represents the sum of squares of the modal amplitudes over the individual damper connections when the undamped mode shape vector is scaled to unit modal mass, i.e. $\mathbf{u}_{0}^{T} \mathbf{M} \mathbf{u}_{0}=1$. With the relation between the filter parameters given as $\omega_{f}=\tau_{f} \omega_{0}^{2}$ the magnitude of the transfer function in (19) can be approximated as

$$
|H(\omega)| \simeq\left|\frac{1}{1-\nu}\right|,
$$

assuming that the angular frequency is sufficiently close to the resonance frequency, i.e. $\omega \simeq \omega_{0}$. Substitution of (34) into (32) gives the optimum damping parameter

$$
c_{o p t} \simeq \frac{2\left(\omega_{\infty}-\omega_{0}\right)}{\gamma^{2}}|1-\nu|,
$$

where the approximation $\left(\omega_{\infty}^{2}-\omega_{0}^{2}\right) / \omega \simeq 2\left(\omega_{\infty}-\omega_{0}\right)$ has been used. The fraction on the right hand side of (35) corresponds to the optimum damping parameter for the pure viscous damper, while the final factor $|1-\nu|$ represents the correction due to the filtered IFF format. By choosing $0<\nu<1$ for increased damper stroke the magnitude of $c_{o p t}$, and thereby the size of the viscous dash-pots, is reduced accordingly. In order for the tuning to be optimal for any orientation of the RNA, the expression in (35) should be independent with respect to the orientation of the RNA for a constant value of $\nu$, and thereby perform identical for both the side-side mode and the fore-aft mode. In figure 5 the total modal damper displacement $\gamma$ defined in (33) is plotted as function of the RNA orientation defined by the angle $\theta_{2}^{1}$ in figure 4c. In the figure 5 the subscript $s s$ refers to the side-side mode, while $f a$ refers to the fore-aft mode. The three different curves are for an increasing number of dampers $r=2$ (dashed), $r=3$ (solid) and $r=5$ (dashdotted). In each configuration the dampers are positioned symmetrically with respect to the circumferential direction, whereby the angle $\Delta \theta_{2}=\theta_{2}^{k+1}-\theta_{2}^{k}$ between two adjacent dampers is given as $\Delta \theta_{2}=360^{\circ} / \mathrm{r}$. The angle $\theta_{2}^{1}$ describes the orientation of the first damper. For two dampers the value for $\gamma$ varies as a function of the RNA orientation, while for three or more dampers $\gamma$ is constant and approximately the same for fore-aft and side-side vibration modes. Table 1 shows the undamped frequencies $\omega_{0}$ and $\omega_{\infty}$, the damping ratio $\zeta_{s t}$ for the two modes, and the optimum damper parameter $c_{\text {opt }}$ for three dampers $(r=3)$. For more than three dampers $\gamma$ is simply increased proportionally according to the number of dampers, whereby the optimum 
damper parameter $c_{o p t}$ is reduced according to (35). In a practical implementation a sufficiently large number of dampers would be preferable because it reduces the size of each damper and it limits the local force impact on the tower wall from the individual damper. In the present beam model all dampers are connected to the same dofs, and the tower therefore only experiences a single damper, representing the combined effect of all dampers through the force vector in (23). To keep the computations as simple as possible only three dampers are included in the beam model. It is found that for three or more dampers in a symmetric circumferential configuration the ratio of the optimum viscous parameter obtained from (35) for the fore-aft (fa) and side-side (ss) vibration modes is $c_{o p t, f a} / c_{o p t, s s}=1.02$. Because this ratio is close to unity the same size viscous dampers and filter parameters can be used for all hybrid dampers inside the tower. For the following simulations the mean value $c_{o p t}=\left(c_{o p t, s s}+c_{o p t, f a}\right) / 2$ is used as the optimum damping parameter, and the corresponding mean frequency $\omega_{0}=\left(\omega_{0, s s}+\omega_{0, f a}\right) / 2$ is used to determine filter parameters for the controller.

\section{DAMPING OF WIND TURBINE TOWER VIBRATIONS}

The performance of the hybrid damper configuration is now investigated. In the first part of this section the wind turbine is assumed at standstill and a root locus analysis, a frequency response analysis and a time transient analysis is conducted. In the final part the performance of the hybrid damper is illustrated by simulations conducted in the commercial aeroelastic code HAWC2 [23]. As discussed in the previous section three hybrid dampers are installed symmetrically inside the tower, connected to the tower bottom and to the tower walls at $4 \mathrm{~m}$ above the bottom. Furthermore, in order for the damping to be independent of the particular orientation of the RNA, the same viscous parameter $c$ and the same controller with filter parameters $\nu, \tau_{f}$ and $\omega_{f}$ are assumed for all three hybrid dampers inside the tower.

4.1. Root locus analysis. The optimum tuning in (35) is based on an assumption that the trajectory of the root of the damped tower mode approximately follows a semicircle in the complex plane from the undamped frequency $\omega_{0}$ to the undamped frequency $\omega_{\infty}$ when the dampers are fully locked [22]. For this idealized semicircular trajectory the corresponding maximum attainable damping ratio is given as

$$
\zeta_{\max }=\frac{\omega_{\infty}-\omega_{0}}{\omega_{\infty}+\omega_{0}} .
$$

With respect to the wind turbine frequencies in Table 1 the predicted maximum attainable damping ratio in (36) becomes $\zeta_{\max }^{s s}=0.0129$ for the side-side mode and $\zeta_{\max }^{f a}=0.0130$ for the fore-aft

TABLE 1. Modal properties for vibration modes and optimum damper values for three dampers with $\nu=0$

\begin{tabular}{lcccc}
\hline Mode & $\omega_{0} /(2 \pi)[\mathrm{Hz}]$ & $\omega_{\infty} /(2 \pi)[\mathrm{Hz}]$ & $\zeta_{\text {st }}[\%]$ & $c_{\text {opt }}[\mathrm{Ns} / \mathrm{m}]$ \\
Side-side & 0.2418 & 0.2481 & 1.15 & $5.910^{9}$ \\
Fore-aft & 0.2437 & 0.2501 & 1.15 & $6.010^{9}$ \\
\hline
\end{tabular}


mode. To investigate the accuracy of the optimum tuning, the wind turbine with hybrid dampers installed is now investigated with respect to a root-locus analysis. The system of governing equations in (27) is conveniently rewritten to state-space format without external loading $\mathbf{f}=\mathbf{0}$,

$$
\frac{d}{d t}\left[\begin{array}{c}
\mathbf{u} \\
\dot{\mathbf{u}} \\
\mathbf{q} \\
\dot{\mathbf{q}}
\end{array}\right]=\left[\begin{array}{cccc}
\mathbf{0}_{u u} & \mathbf{I}_{u u} & \mathbf{0}_{u q} & \mathbf{0}_{u q} \\
-\mathbf{M}^{-1} \mathbf{K} & -\mathbf{M}^{-1}\left(\mathbf{C}+c \mathbf{W} \mathbf{W}^{T}\right) & \mathbf{0}_{u q} & c \mathbf{M}^{-1} \mathbf{W} \\
\mathbf{0}_{u q}^{T} & \mathbf{0}_{u q}^{T} & \mathbf{0}_{q q} & \mathbf{I}_{q q} \\
\mathbf{0}_{u q}^{T} & -\nu / \tau_{f} \mathbf{W}^{T} & -\omega_{f} / \tau_{f} \mathbf{I}_{q q} & -(1-\nu) / \tau_{f} \mathbf{I}_{q q}
\end{array}\right]\left[\begin{array}{c}
\mathbf{u} \\
\dot{\mathbf{u}} \\
\mathbf{q} \\
\dot{\mathbf{q}}
\end{array}\right],
$$

where $\mathbf{0}$ and $\mathbf{I}$ denote the zero and identity matrices, respectively, with subscripts $u$ and $q$ identifying the specific dimensions with reference to the number of components in the corresponding vectors $\mathbf{u}$ and $\mathbf{q}$. The complex valued natural frequencies $\omega$ are determined directly from the eigenvalues $i \omega$ of the system matrix in (37). The natural frequency can be written as

$$
\omega=|\omega|\left(\sqrt{1-\zeta^{2}}+i \zeta\right)
$$

where the corresponding damping ratio is computed as

$$
\zeta=\frac{\operatorname{Im}[\omega]}{|\omega|} .
$$

Thus, the attainable damping ratio is seen to be closely related to the maximum of the imaginary part of the complex frequency.

Figure $6(\mathrm{a}, \mathrm{b})$ shows the trajectories of the complex natural frequency of the side-side and fore-aft vibration modes for an increasing value of the damping parameter $c$, a so-called root locus. The solid black curve is for $\nu=0$ and therefore represents the pure viscous case without actuator motion. The dashed black curve represents proportional intrinsic damping $\zeta_{s t}=0.0115$, with contributions from structural and soil damping as introduced in Section 3.1. Because the wind turbine is analyzed at standstill aerodynamic damping is omitted in this initial analysis. The remaining three curves in the figure are for $\nu=0.9$ with $\omega_{f}=\tau_{f} \omega_{0}^{2}=\omega_{0} / 8$ (blue), for $\nu=0.9$ with $\omega_{f}=\tau_{f} \omega_{0}^{2}=\omega_{0}$ (red) and for $\nu=0.975$ with $\omega_{f}=\tau_{f} \omega_{0}^{2}=\omega_{0} / 8$ (green). The natural frequencies obtained from the eigenvalue analysis for $c_{o p t}$ in (35) are represented by the blue circles in the figure. The corresponding damping ratio determined by (39) is shown in figure $6(\mathrm{c}, \mathrm{d})$. The proportional damping and the supplemental damping from the hybrid dampers are found to be approximately additive. For the pure viscous case (black solid) the trajectory accurately reproduces the expected semicircle, and the damping ratio at $c_{\text {opt }}$ reproduces the estimated total maximum damping $\zeta_{t o t} \simeq \zeta_{s t}+\zeta_{\max } \simeq 0.245$. For increasing values of both the filter parameters and the gain value the semicircular shape is distorted and the attainable damping is consequently reduced. As seen from the damping ratio in figure $6(\mathrm{c}, \mathrm{d})$ an increase in the filter and gain parameters implies that $c_{\text {opt }}$ overestimates the actual optimum. The present results indicate that for gains $\nu \leq 0.9$ and filter parameters $\omega_{f}=\tau_{f} \omega_{0}^{2}<\omega_{0}$ the complex trajectories approximately reproduces the desired semicircle of the pure viscous case and $c_{\text {opt }}$ gives a good estimate of the optimum damping parameter. 
(a)

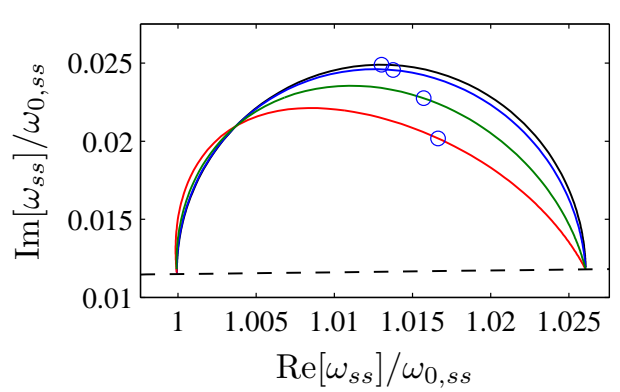

(c)

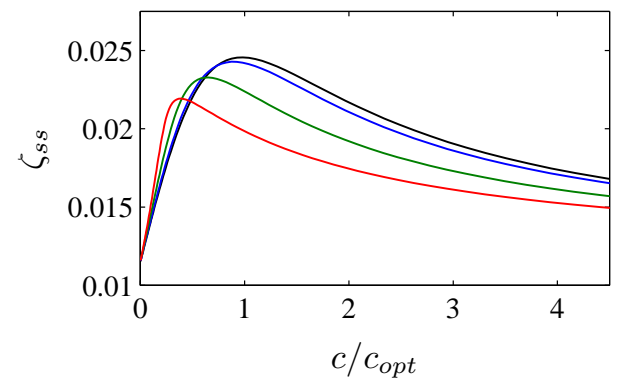

(b)

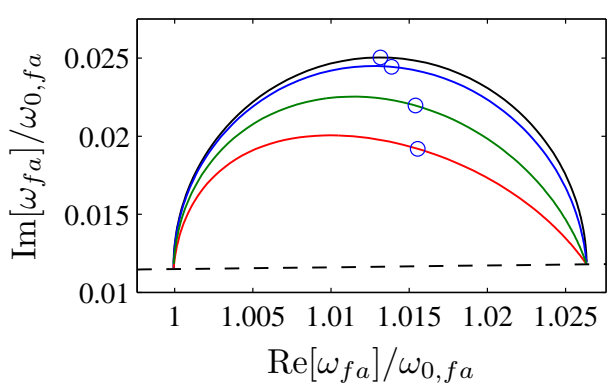

(d)

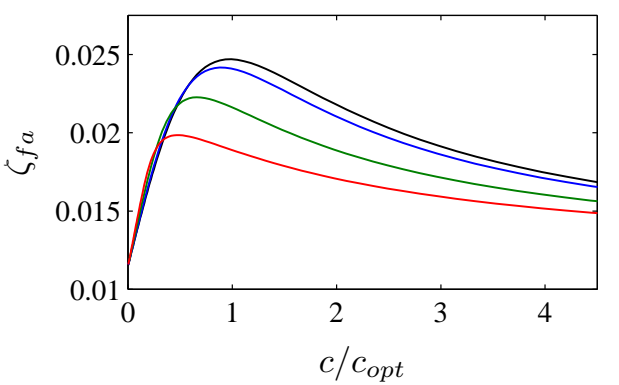

FiguRE 6. (a,b) Root locus plot and (c,d) damping ratio for increasing $c$, for side-side mode in $(\mathrm{a}, \mathrm{c})$ and fore-aft mode in $(\mathrm{b}, \mathrm{d})$. Damper parameters: $\omega_{f}=$ $\tau_{f} \omega_{0}^{2}=\omega_{0} / 8$ with $\nu=0$ (black), $\nu=0.9$ (blue) and $\nu=0.975$ (green), and $\omega_{f}=\tau_{f} \omega_{0}^{2}=\omega_{0}$ with $\nu=0.9$ (red).

4.2. Frequency response analysis. In this section the hybrid damper concept is investigated by a frequency response analysis, assuming harmonic solutions of the form

$$
\mathbf{u}=\overline{\mathbf{u}} \exp i \omega t \quad, \quad q=\bar{q} \exp i \omega t \quad, \quad \mathbf{f}=\overline{\mathbf{f}} \exp i \omega t
$$

with amplitudes denoted by a bar and with driving angular frequency $\omega$. Substituting (40) into the governing equations in (27) gives

$$
\left(-\omega^{2}\left[\begin{array}{cc}
\mathbf{M} & \mathbf{0} \\
\mathbf{0}^{T} & \tau_{f} \mathbf{I}
\end{array}\right]+i \omega\left[\begin{array}{cc}
\mathbf{C}+c \mathbf{W} \mathbf{W}^{T} & -c \mathbf{W} \\
\nu \mathbf{W}^{T} & (1-\nu) \mathbf{I}
\end{array}\right]+\left[\begin{array}{cc}
\mathbf{K} & \mathbf{0} \\
\mathbf{0}^{T} & \omega_{f} \mathbf{I}
\end{array}\right]\right)\left[\begin{array}{c}
\overline{\mathbf{u}} \\
\overline{\mathbf{q}}
\end{array}\right]=\left[\begin{array}{c}
\overline{\mathbf{f}} \\
\mathbf{0}
\end{array}\right],
$$

from which the structural amplitudes $\overline{\mathbf{u}}$ and actuator amplitudes $\overline{\mathbf{q}}$ are determined for a given load amplitude $\overline{\mathbf{f}}$. The damper amplitudes $\bar{u}_{k}$ are determined subsequently by (24),

$$
\bar{u}_{k}=\mathbf{w}_{k}^{T} \overline{\mathbf{u}} .
$$

The wind turbine is loaded locally by a single force at Mean Sea Level (MSL) acting at an angle of $45^{\circ}$ relative to the plane of the rotor, hereby representing a wave load misalignment with respect to the wind direction. The force vector can therefore be written as

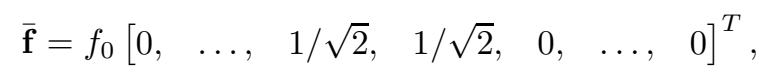

where $f_{0}$ represents the magnitude of the external load.

Figure 7 shows the frequency response of the wind turbine and the hybrid damper for different gain values $\nu=0$ (black), $\nu=0.5$ (red) and $\nu=0.75$ (blue) and with filter parameters $\omega_{f}=$ $\tau_{f} \omega_{0}^{2}=\omega_{0} / 8$. The curves are scaled by the corresponding static displacement $u_{t o p}^{0}$ at the top of the wind turbine tower, whereby figure 7 (a) shows the dynamic amplification of the tower 
(a)

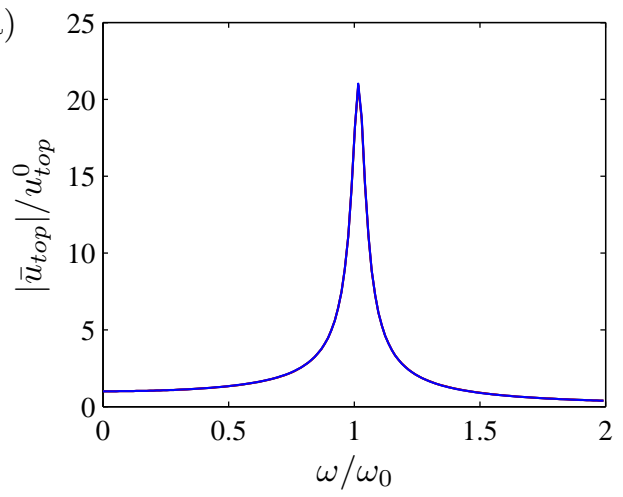

(b) 0.1

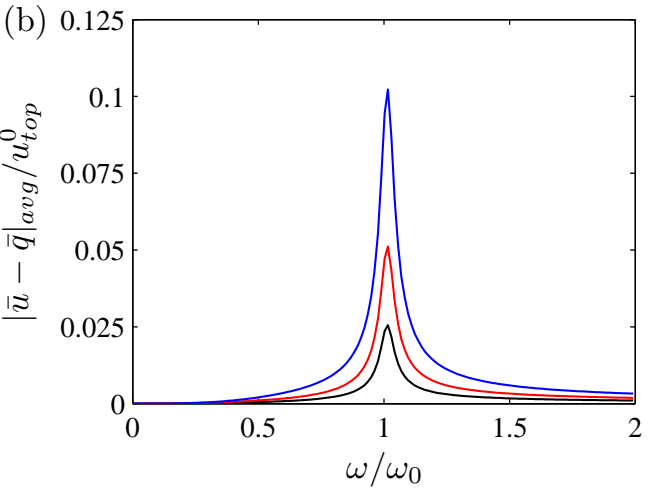

FiguRE 7. (a) Top floor and (b) damper displacement for $\omega_{f}=\tau_{f} \omega_{0}=\omega_{0} / 8$ with $\nu=0.0$ (black), 0.5 (red) and 0.75 (blue).

top. Since the natural frequency and damping of the two tower modes are almost identical, the response of the two modes will be in phase, and therefore only the total response of the tower top is plotted in figure 7(a), and as expected the amplitude is the same for all three values for $\nu$. When the response is represented entirely by a single mode, the dynamic amplification factor at the resonance frequency is $1 /\left(2 \zeta_{t o t}\right)$. Assuming that the response of the wind turbine is a linear combination of the two tower modes this can be used to estimate the damping ratio of the two modes with the hybrid damper installed inside the tower. From the dynamic amplification of the tower top displacement in figure 7 (a) the damping ratio is estimated to be $\zeta_{t o t}=0.0238$, which corresponds fairly well with the damping ratio $\zeta_{\text {tot }}=0.0245$ predicted by the root loci in figure 6 . Figure 7(b) shows the average of the individual displacement amplitudes over the three dampers, which is constant for any orientation of the RNA, as shown in figure 5. It is seen that the damper displacement in figure 7(b) is increased significantly as $\nu \rightarrow 1$, which is in agreement with the expression in (10).

4.3. Time transient analysis and actuator drift. Drift of the actuator displacement may arise due to errors in the force measurements. An error in the force measurement will for the IFF controller correspond to adding an additional force term $f_{e}$ on the right-hand side of equation (2), which by insertion of the damper force from (1) takes the form

$$
\dot{q}(t)=-\nu(\dot{u}(t)-\dot{q}(t))-g f_{e}(t) .
$$

The actuator displacement at time $t_{1}$ can be determined by time integration as

$$
q\left(t_{1}\right)=-\frac{\nu}{1-\nu} u\left(t_{1}\right)-\frac{g}{1-\nu} \int_{0}^{t_{1}} f_{e}(t) d t .
$$

The force error $f_{e}$ is seen to lead to drift of the actuator displacement, and in particular a constant force error will lead to constant increase in the drift. If the drift leads to saturation of the actuator signal, this may cause a significant reduction in the performance of the hybrid damper. The filtered time integration presented in Section 2.2 is introduced in order to avoid drift in the actuator signal, due to errors in the measured force feedback signal. To investigate the effect of the filtered integration the hybrid damper concept is now analyzed by solving the closed-loop 
system of equation in (27) to obtain the time transient response when the wind turbine is loaded by a wave load at MSL. Furthermore, a force offset is included to represent a signal error locally in the feedback control loop of the third hybrid damper. The load consists of a wave train of three regular sine waves with realistic wave period $T_{w}=10 \mathrm{~s}$, whereafter the force drops to zero. The period $T_{w}$ corresponds approximately to 0.4 wave periods per vibration period of the wind turbine. As in the previous example the misaligned wave load acts at an angle of $45^{\circ}$ relative to the rotor direction. The load vector is therefore given as

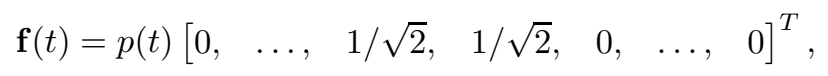

where the time dependence of the load intensity $p(t)$ is introduced as

$$
\frac{p(t)}{f_{0}}=\left\{\begin{array}{cc}
\sin \left(2 \pi t / T_{w}\right) & , \quad t \leq 3 T_{w} \\
0 & , \quad t>3 T_{w}
\end{array}\right.
$$

where $f_{0}$ is the force amplitude. The constant offset error signal $f_{e}=f_{0} / 200$ is included in the feedback signal to the force feedback control of the third damper by simply adding the force term $-g f_{e}$ to the right hand side of the bottom equation in (27). The force error is only applied during the first three wave periods $3 T_{w}$ when the above wave train passes the wind turbine. For $t>3 T_{w}$ the offset error in the force signal is removed, i.e. $f_{e}=0$.

Figure 8 shows the time dependent response of the hybrid damper and wind turbine for $\nu=0.5$ and $\omega_{f}=\tau_{f}=0.0$ with error (blue) and without error (black) in the feedback signal, and for $\omega_{f}=\tau_{f} \omega_{0}^{2}=\omega_{0} / 8$ with error in the feedback signal (red). In figure 8(a) the total displacement of the tower top is plotted. The response of the three simulations are seen to be practically identical and by use of an exponential fit (indicated by the dashed curve) the apparent damping ratio is estimated to be $\zeta_{t o t}=0.0248$. This is in agreement with the damping ratios determined from both the root locus analysis in Section 4.1 and the frequency response analysis in Section 4.2. The actuator displacement of the third hybrid damper $q^{(3)}$ is plotted in figure $8(\mathrm{~b})$. The actuator displacement is seen to have a linear drift during the wave train when an error is included in the feedback signal (blue) compared to the reference case with no errors in the signal (black). After the wave train has passed and the error signal is removed the offset in $q^{(3)}$ is constant. For the case with an error in the feedback signal and filtered integration (red) the offset of the actuator displacement is significantly reduced. Figure 8(c) shows the displacement of the viscous dash-pot for the third hybrid damper $(u-q)^{(3)}$. The amplitude across the viscous dash-pot is amplified to approximately twice the amplitude of the actuator displacement for the gain $\nu=0.5$. Finally, figure $8(\mathrm{~d})$ shows the three damper force histories for the third hybrid damper $f^{(3)}$ with error signal. As expected the damper force is very similar for all the three simulations, and therefore not influenced by the drift in the actuator motion. In the first part of the simulation, when the wind turbine is vibrating in a forced response, the damper force amplitude is small compared to the displacement response of the wind turbine in figure 8(a) and it is of more irregular type. In the subsequent free vibration part the damper force immediately increases, followed by a harmonically decaying response. 
(a)
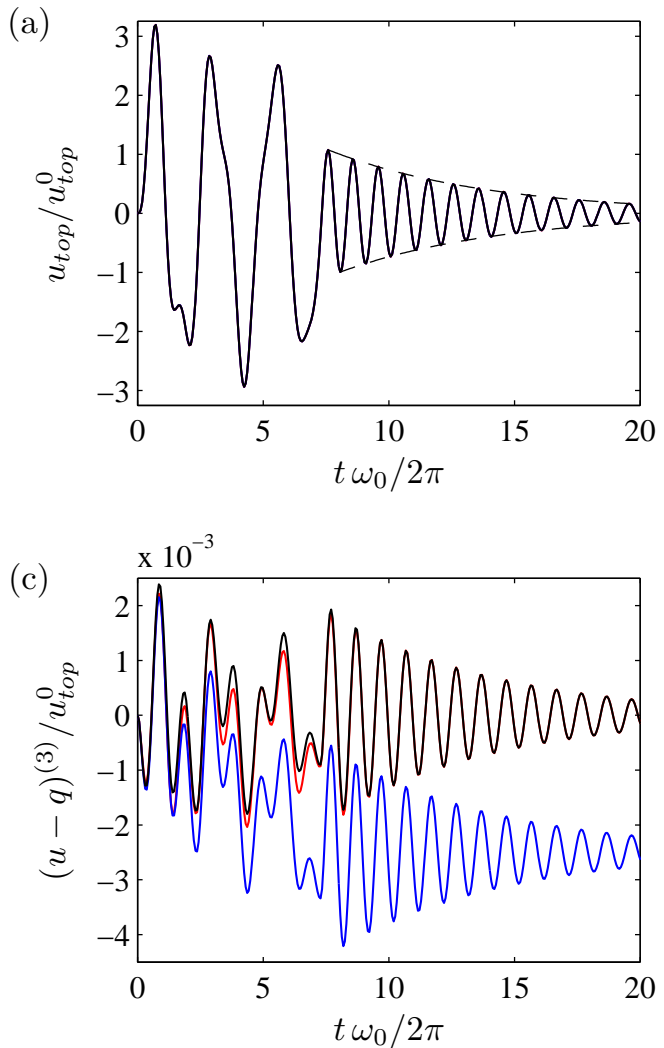

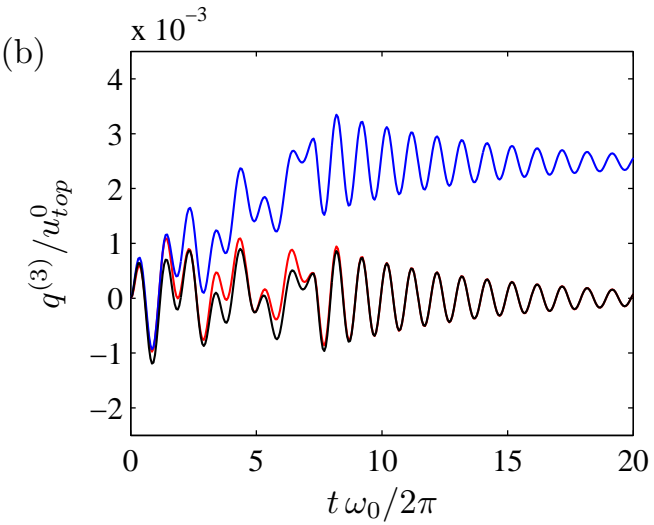

(d)

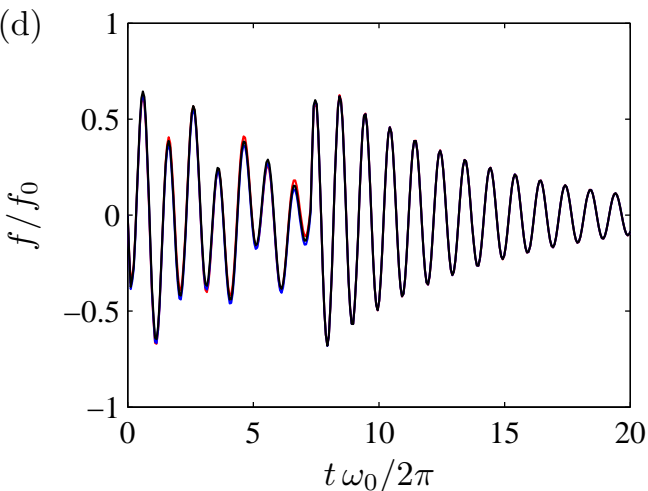

Figure 8. (a) Tower top displacement, (b) actuator displacement, (c) displacement of viscous dash-pot and (d) damper force. The three curves represent: $\nu=0.5$ and $\omega_{f}=\tau_{f}=0.0$ with error feedback signal (blue) and without error feedback signal (black), and with $\omega_{f}=\tau_{f} \omega_{0}^{2}=0.125$ and error feedback signal (red).

4.4. Time transient simulations in HAWC2. Aerodynamic damping is the dominant damping component for the fore-aft vibrations in the rotor direction ( $y$-axis) during operations of a wind turbine, while the associated side-side vibrations in the rotor plane direction ( $x$-axis) are very lightly damped and therefore the target of the hybrid damper system. In the previous analysis, where the wind turbine is modeled by simple beam elements, the aerodynamic effects are omitted. To investigate the effect of aerodynamic damping the time response simulations are now carried out using the dedicated aeroelastic code HAWC2 (Horizontal Axis Wind turbine simulation Code 2nd generation), introduced in the user manual [23]. The HAWC2 code is developed at DTU Wind Energy and used both for industrial applications and research projects. The code includes a multibody formulation for modeling of the nonlinear structural dynamics and a so-called Stig Øye model for the aerodynamic modeling of the blades. Thus, the use of HAWC2 allows for very realistic dynamic simulations of wind turbines during operations and not only at standstill, as in the previous analysis in this section. The model used for the simulations is downloaded from the HAWC2 webpage [24], and this model is equivalent to that used in the OC3-study [19]. 

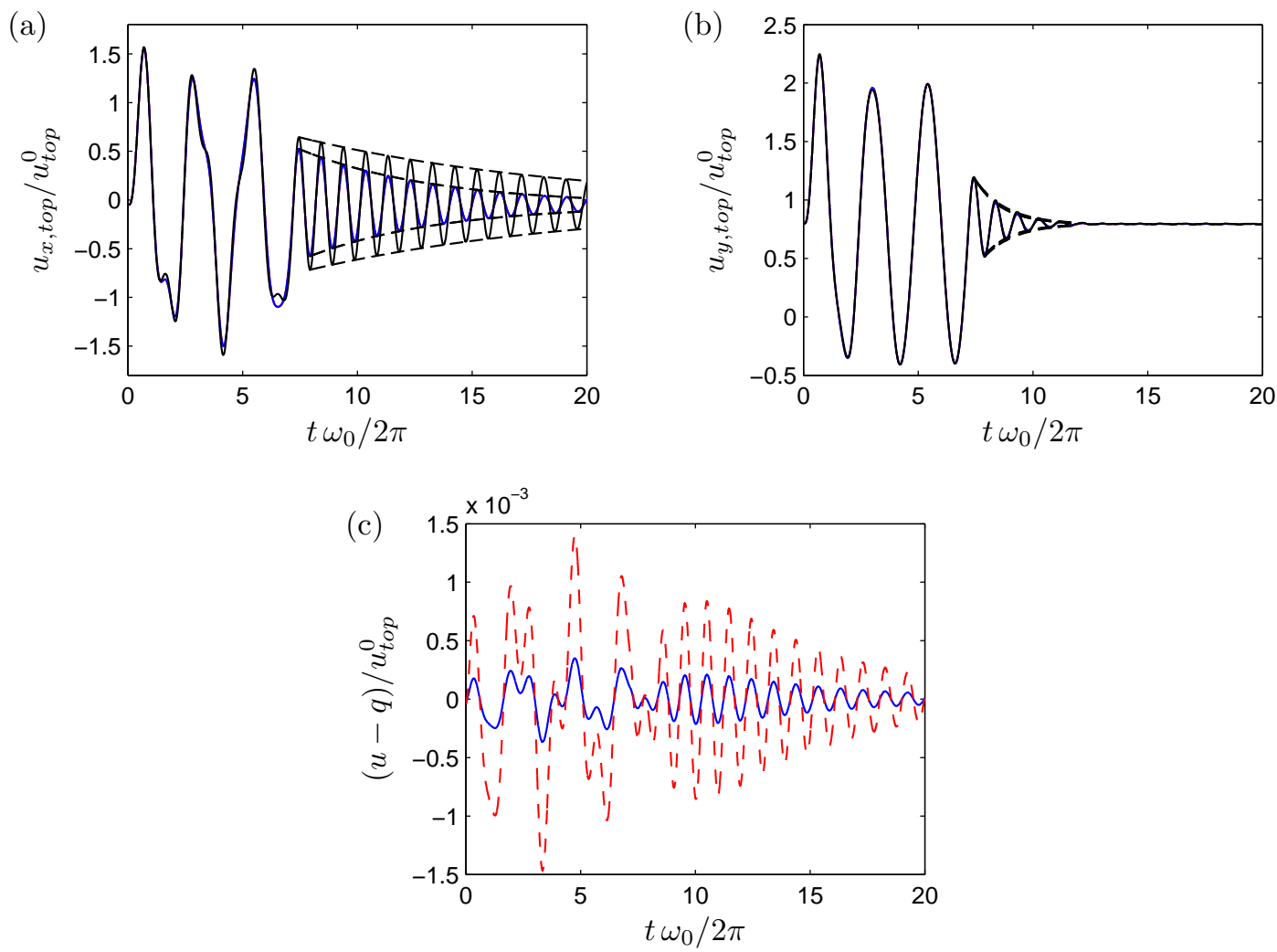

Figure 9. Tower top displacement in $x$-direction (a) and $y$-direction (b), and (c) displacement over the viscous dash-pot for $\nu=0$ (blue) and for $\nu=0.75$ (red) with $\omega_{f}=\tau_{f} \omega_{0}^{2}=\omega_{0} / 8$ and without dampers (black).

The wind turbine is loaded by a mean wind speed of $8 \mathrm{~m} / \mathrm{s}$, with zero turbulence intensity and a wind shear profile with power law exponent 0.14. The aerodynamic forces on the blades are computed using a Blade Element Momentum model and aerodynamic drag on the tower and nacelle is included as well. Soil and structural damping is tuned to give a critical damping ratio of $\zeta_{s t}=0.0115$ for the two lowest tower (side-side and fore-aft) modes, which is identical to the linear beam model used in the previous part of this section. In HAWC2 the hybrid dampers are effectively implemented using an external dynamic link library (dll), as explained in [25] for an advanced dynamic gear model. In addition to the wind load the wind turbine is also loaded by a wave train identical to the wave loading in the previous example, whereby the fore-aft mode and the side-side mode are simultaneously excited with equal magnitude. When increasing the misalignment of the wave train relative to the wind direction the excitation of the lightly damped side-side mode will increase as well, resulting in larger vibration amplitudes. Thus, the magnitude of the displacement amplitudes presented in this section depends on the specific angle of misalignment because of the large aerodynamic damping in the fore-aft direction. The wave train is applied sufficiently long time after simulation startup so that any initial transient response from the wind load has disappeared. Simulations are conducted for three cases: (a) without dampers, (b) with three viscous dampers and (c) with three hybrid dampers installed inside the tower. For 
the hybrid dampers the gain parameter $\nu=0.75$ and the filter parameters $\omega_{f}=\tau_{f} \omega_{0}^{2}=\omega_{0} / 8$ have been used.

In the HAWC2 model the $x$-direction represents the in-plane direction and is therefore associated with the lightly damped side-side vibrations, while the corresponding $y$-direction represents the fore-aft vibration form. As seen in figure $9(\mathrm{a}, \mathrm{b})$ vibrations in the $y$-direction are damped much faster than the corresponding vibrations in the $x$-direction. This is due to the additional aerodynamic damping in the rotor direction. Without dampers the effective damping ratio for the fore-aft vibrations is estimated from vibration decay to $\zeta_{\text {tot }}=0.1123$, while this value increases to $\zeta_{\text {tot }}=0.1267$ in the case with hybrid or viscous dampers. These values are significantly higher than the values from the previous standstill simulations due to the presence of aerodynamic damping. For the side-side vibrations the damping ratio is similarly estimated to $\zeta_{t o t}=0.0122$ for the case without supplemental dampers, while it increases to $\zeta_{\text {tot }}=0.0264$ with dampers, which is slightly larger than the values obtained previously from the simulations at standstill. This increase could be due to modal interaction with the fore-aft mode, which has a significantly larger modal damping.

The additional damping from the supplemental dampers is obtained from the total damping ratios, by subtracting the values obtained from the simulations without dampers. This gives additional damping ratios of $\zeta_{f a}=0.0144$ for the fore-aft vibrations and $\zeta_{s s}=0.0142$ for the side-side vibrations. These values are slightly larger then the expected value $\zeta_{\max }=0.013$ obtained from the previous analysis. These higher damping values are likely due to changes in the vibration form of the structure during operation caused by the insertion of dampers at the bottom of the tower. The modified vibration form causes increased soil and aerodynamic damping, which is added to the additional damping from the supplemental dampers. Thus, the present simulations conducted in HAWC2 show that the performance of the hybrid damper concept is unaffected by aerodynamic damping, and that inherent and supplemental damping values are approximately additive, also during operation of the wind turbine. Finally, in figure 9 (c) the displacement over the viscous damper element in the hybrid damper is plotted. When comparing the pure viscous case with the hybrid damper using $\nu=0.75$ the displacement across the damper is amplified by approximately a factor of four, which again agrees with the theoretical predictions from the expression in (10).

4.5. Fatigue analysis in HAWC2. A three hour wave load record is now prepared and applied to the off-shore wind turbine in HAWC2. The wind conditions are identical to those for the transient analysis in the previous Section 4.4, which means that the wind is without turbulence and therefore only introduces a wind pressure in the $y$-direction and aerodynamic effects. The wave loading is generated as a three hour $(10800 \mathrm{~s})$ realization of a common JONSWAP spectrum, with a peak frequency of $0.25 \mathrm{~Hz}$ and peak factor 3.3, see details in [19]. The weave load is again applied with a misalignment angle of $45^{\circ}$ relative to the $x$-axis, whereby the fore-aft and the sideside directions receive the same force components. Thus, the wave loading introduces the only stochastic loading on the structure and the reduction of the extreme response and accumulated 
damage due to the presence of the active damper system is investigated in this section. The simulations in HAWC2 with and without damper system are conducted for the same seed in the realization of the wave loading, whereby the time histories for the damped response are directly comparable to those without damper system.

Figure 10 shows the time response histories of (a) the top tower position $u_{x, \text { top }}$ in side-side $x$ direction and the base bending moment $M_{y, \text { base }}$ around the $y$-axis at the bottom of the tower. The time $t$ is again normalized by the natural frequency $\omega_{0} /(2 \pi)=0.2418 \mathrm{~Hz}$, and it is seen that the length of the time records in the figure correspond to around 2600 vibration periods. The response of the wind turbine without damper is represented by the gray curve in the background, while the red curve in the front shows the damped response with $\nu=0.75$ and $\omega_{f}=\tau_{f} \omega_{0}^{2}=\omega_{0} / 8$. The black dashed horizontal line indicates the (common) mean value, while the gray and red dashed lines represent the corresponding standard deviations. The maximum deflections and bending moments are indicated in the figure by the asterisk markers. It is seen that the top tower deflection is only reduced slightly by the hybrid damper system, with a reduction in maximum deflection (relative to mean) of $13 \%$ and a corresponding reduction in standard deviation of $23 \%$. The reduction in the base moment at the bottom of the tower is significantly larger. The maximum bending moment (relative to mean) is reduced by $38 \%$ due to presence of the hybrid damper system, while the standard deviation of the base moment is reduced by $49 \%$.
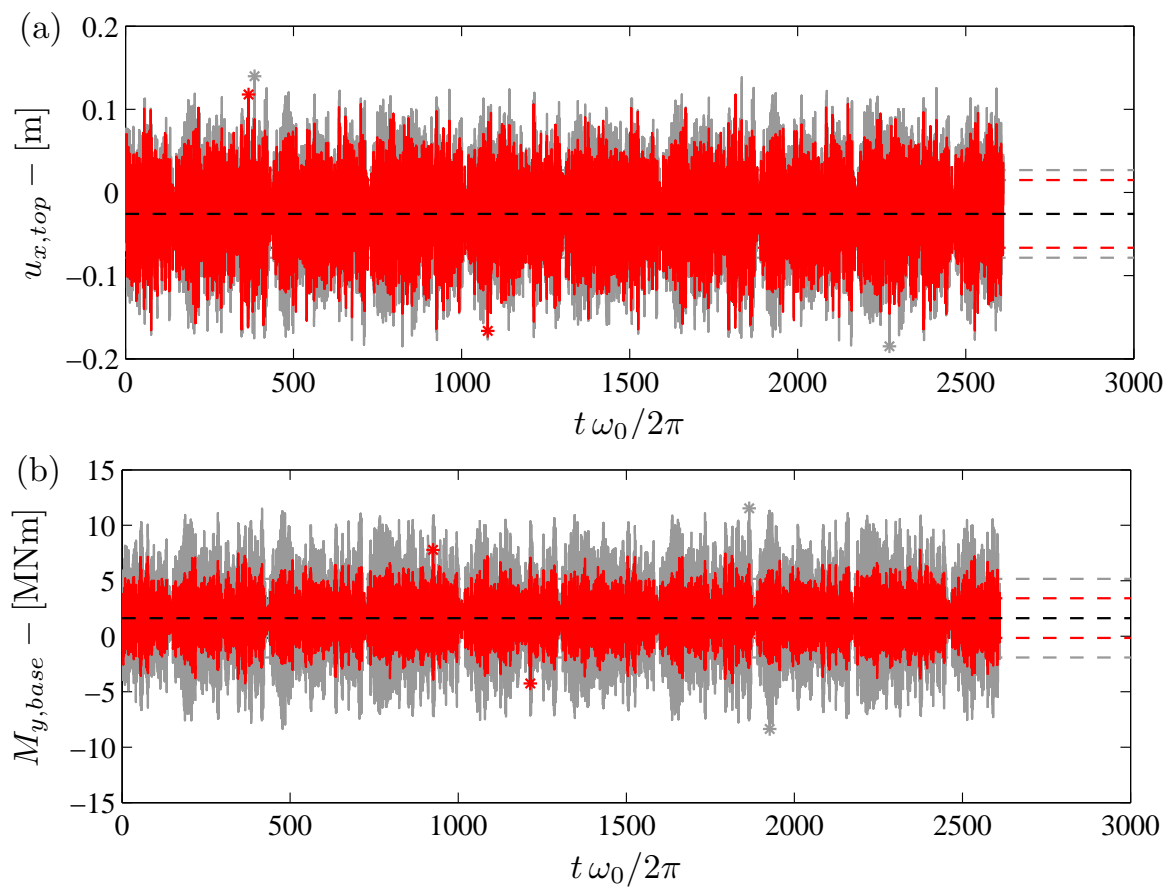

Figure 10. Tower top position in side-side $x$-direction (a) and base moment around $y$-axis (b). 
A fatigue analysis is conducted based on the irregular response of the wind turbine tower using the rainflow counting procedure described in [26]. Typically (normal) stress cycles are detected and accumulated. However, in the present case the base bending moment $M_{y, \text { base }}$ is used directly in the fatigue analysis. A standard rainflow counting procedure is applied to generate moment increments $\Delta M_{j}$ from the base bending moment record associated. The accumulated damage $D_{M}$ is then estimated from the summation

$$
D=\sum_{j=1}^{N} \frac{\Delta M_{j}^{m}}{K}
$$

with power law exponent $m$ and constant $K$, see [26]. The present results for the damage are normalized by the constant $K$ and an exponent of $m=3$ is assumed. In the case without damper the rainflow counting procedure detects $N_{0}=3475$ cycles, while in the damped case this number is reduced to $N_{d}=2313$, which indicates that the damped response is significantly more regular than the undamped case. The accumulate damage without damper system is denoted $D_{0}$, while $D_{d}$ in the damped case. The accumulated damage ratio is obtained as $D_{d} / D_{0}=0.13$, while a corresponding damage ratio, corrected with respect to the individual number of cycles, is determined as $\left(D_{d} N_{0}\right) /\left(D_{0} N_{d}\right)=0.20$. Both damage ratios indicate that the hybrid damper system proposed in the present paper is able to reduce the accumulate damage and thereby increase the fatigue life of the off-shore wind turbine.

\section{Conclusion}

Damping of wind turbine tower vibrations due to misaligned wave loading is expected to be critical for the feasibility of the monopile support structure to be used for future offshore wind turbines at larger water depths. The present paper presents a novel solution for damping of tower vibrations, where viscous hybrid dampers are installed at the bottom of the wind turbine tower. In the hybrid damper concept the actuator uses an Integral Force Feedback (IFF) scheme for amplifying the displacement over the viscous dash-pot element, and thereby significantly increasing the feasibility of installing dampers at the bottom of the tower. In this pure IFF format attainable damping is fully equivalent to the attainable damping of a passive viscous dash-pot, and the optimum damping parameter may therefore be determined by appropriate scaling of the corresponding optimum damping parameter for the pure passive case obtained by the results in [22]. To avoid drift in the actuator displacement, for example due to errors in the force measurements, a filtered integration of the actuator velocity is introduced. It is demonstrated through numerical examples that this filtering can significantly reduce the drift without notable loss of the damping performance. For large values of the filter parameters or gain values in the IFF controller a reduction in attainable damping is found, which therefore limits the potential amplification of the damper displacement. It is found that with filter parameter values $\omega_{f}=\tau_{f} \omega_{0}^{2}=\omega_{0} / 8$ and gain values $\nu=0.5-0.9$ the associated amplification factor ranges from 2 to 10, while the attainable damping is approximately similar to that obtained by the pure passive viscous case. In the numerical simulations the $5 \mathrm{MW}$ offshore reference wind turbine from the Offshore Code Comparison Collaboration study [19] is 
considered. For this model it is demonstrated that when several hybrid dampers are installed in a symmetric circumferential layout the same size of dampers and filter parameters for each damper may be used without significant decrease in performance. Thus, the present damping configuration is sufficiently independent of the direction of the Rotor Nacelle Assembly. Furthermore, the simulations show that by placing hybrid dampers in a symmetric layout connected to the tower wall at the bottom of the tower and 4 meters above the bottom a damping ratio of approximately $\zeta=0.0125$ is achievable in both of the two lowest critical tower modes. Finally, a fatigue analysis is conducted in HAWC2, using irregular wave loading, which shows that the hybrid damper system reduces the accumulated damage in the wind turbine tower. The damping of the fore-aft and sideside vibration modes of the wind turbine might also affect other important vibration problems. For example, the present concept might introduce supplemental damping to the lightly damped edgewise vibration modes of the rotor, which are coupled to the side-side tower motions, see [27]. Furthermore, the present approach could also be effective in damping of the vibrations induced by voltage sag due to for example grid fault, see [28, 29].

Acknowledgments. This work has been supported by the Danish Energy Agency and Vestas Wind Systems A/S under the EUDP project 'Monopile cost reduction and demonstration by joint applied research'.

\section{REFERENCES}

[1] Niels Jacob Tarp-Johansen, L Andersen, E. D. Christensen, C Mørch, B Kallesøe, and S Frandsen. Comparing sources of damping of cross-wind motion. In Proceedings of the European Wind Energy Conference \& Exhibition, Stockholm, Sweden, 2009.

[2] Mads Damgaard, L. B. Ibsen, L. V. Andersen, and J. K. F. Andersen. Cross-wind modal properties of offshore wind turbines identified by full scale testing. Journal of Wind Engineering and Industrial Aerodynamics, 116:94-108, 2013.

[3] P. J. Murtagh, A. Ghosh, B. Basu, and B. M. Broderick. Passive control of wind turbine vibrations including blade/tower interaction and rotationally sampled turbulence. Wind energy, 11:305-317, 2008.

[4] S Colwell and B Basu. Tuned liquid column dampers in offshore wind turbines for structural control. Engineering Structures, 31(2):358-368, 2009.

[5] Matthew A. Lackner and Mario A. Rotea. Passive structural control of offshore wind turbines. Wind energy, 14(3):373-388, 2011.

[6] Gordon M Stewart and Matthew A Lackner. The impact of passive tuned mass dampers and wind-wave misalignment on offshore wind turbine loads. Engineering Structures, 73:54-61, 2014.

[7] V. N. Dinh and B. Basu. Passive control of floating offshore wind turbine nacelle and spar vibrations by multiple tuned mass dampers. Structural Control and Health Monitoring, 22:152$176,2015$. 
[8] Karl A Stol, Wenxin Zhao, and Alan D Wright. Individual blade pitch control for the Controls Advanced Research Turbine (CART). Journal of solar energy engineering, 128(4):498-505, 2006.

[9] T. Fischer, P. Rainey, E. Bossanyi, and M. Kühn. Study on control concepts suitable for mitigation of loads from misaligned wind and waves on offshore wind turbines supported on monopiles. Wind Engineering, 35(5):561-574, 2011.

[10] ML Brodersen and J Høgsberg. Damping of offshore wind turbine tower vibrations by a stroke amplifying brace. Energy Procedia, 53.

[11] Arturo Rodrguez Tsouroukdissian. System and method for damping vibrations in a wind turbine. US Patent 8,641,369, 2014.

[12] J Høgsberg and ML Brodersen. Hybrid viscous damper with filtered integral force feedback control. Journal of Vibration and Control, 2014.

[13] A Preumont, JP Dufour, and C Malekian. Active damping by a local force feedback with piezoelectric actuators. Journal of guidance, control, and dynamics, 15(2):390-395, 1992.

[14] Andre Preumont. Vibration Control of Active Structures: An Introduction. Third edition. Springer Verlag, Berlin, 2011.

[15] Y. Achkire and A. Preumont. Active tendon control of cable-stayed bridges. Earthquake Engineering \& Structural Dynamics, 25(6).

[16] Frédéric Bossens and André Preumont. Active tendon control of cable-stayed bridges: a largescale demonstration. Earthquake engineering \& structural dynamics, 30(7):961-979, 2001.

[17] André Preumont, Younes Achkire, and Frédéric Bossens. Active tendon control of large trusses. AIAA journal, 38(3):493-498, 2000.

[18] JR Høgsberg and S Krenk. Linear control strategies for damping of flexible structures. Journal of Sound and Vibration, 293(1):59-77, 2006.

[19] J Jonkman and W Musial. Offshore code comparison collaboration (OC3) for IEA task 23 offshore wind technology and deployment. Technical report, National Renewable Energy Laboratory, 2010. NREL/TP-500-48191.

[20] S Krenk. A general format for curved and non-homogeneous beam elements. Computers 83 structures, 50(4):449-454, 1994.

[21] Wolfgang Hahn and Arne P Baartz. Stability of motion, volume 422. Springer, 1967.

[22] JA Main and S Krenk. Efficiency and tuning of viscous dampers on discrete systems. Journal of Sound and Vibration, 286(1-2):97-122, 2005.

[23] Torben J Larsen and Anders Melchior Hansen. How 2 HAWC2, the user's manual. Ris $\varnothing$ National Laboratory, 2007.

[24] Phase II, extented monopile. http://www.hawc2.dk/HAWC2\%20Download/HAWC2\%20model.aspx. Accessed: 04-01-2015.

[25] A. M. Hansen and T. J. Larsen. Gear dynamics. Technical report, Risoe National Lab., 2009. Research in Aeroelasticity EFP-2007-II, Risoe-R-1698(EN):134-142.

[26] Henrik O. Madsen, Steen Krenk, and Niels C. Lind. Methods of Structural Safety. Dover Publications, New York, 2006. 
[27] Morten H. Hansen. Aeroelastic instability problems for wind turbines. Wind energy, 10:551$577,2007$.

[28] R. Fadaeinedjad, G. Moschopoulos, and M. Moallem. Investigation of voltage sag impact on wind turbine tower vibrations. Wind energy, 11:351-375, 2008.

[29] Biswajit Basu, Andrea Staino, and Malabika Basu. Role of flexible alternating current transmission systems devices in mitigating grid fault-induced vibration of wind turbines. Wind energy, 17:1017-1033, 2014. 